\title{
La importancia e influencia del idioma inglés dentro del campo científico
}

\section{The importance and influence of the English language within the scientific field}

\author{
Giovanni Beltrán-Santoyo ${ }^{a}$, Esther A. Ruíz-Huerta ${ }^{b}$, Juan M. $^{c}$ Gómez-Bernal
}

\begin{abstract}
:
Through the years, the English language has evolved and dominated many forms of communication which has allowed it to emerge as one of the most important languages around the world, period it has generated a great impact on the world and has served as a fundamental tool for communication internationally, since it has facilitated society to have greater communication, but most of all, in the field of science, an international development has been generated. Even though English is not mother tongue, with the advancement of technology, the number of non-native people who speak English has increased, allowing them to publish their most recent findings around the world. Therefore, this review compiles information to publish the impact that the English language has generated at an international level in the field of science. It was found that nowadays there are different tools and strategies that facilitate the process of writing/publishing in English an appropriate way.
\end{abstract}

Keywords:

Impact, science, communication, translation, grammar

\section{Resumen:}

A través de los años, el inglés ha ido evolucionando y dominando las diversas formas de comunicación, lo cual ha permitido que surja como uno de los idiomas más importantes alrededor del mundo. Ha generado un gran impacto en todo el mundo y ha servido como herramienta fundamental para la comunicación de manera internacional, ya que ha facilitado que la sociedad tenga una mayor comunicación, pero sobre todo, que en el campo de la ciencia se haya logrado generar una expansión de manera global. A pesar de que la mayoría de los países no tienen el inglés como lengua materna, con el avance de la tecnología ha aumentado el número de personas no nativas que dominan el idioma inglés y pueden publicar sus hallazgos más recientes alrededor del mundo. Es por eso, que esta revisión recopila información para dar a conocer el impacto que ha generado el idioma inglés a nivel internacional en el campo de la ciencia. Se encontró que hoy en día existen diversas herramientas y estrategias que nos facilitan la redacción en inglés de una manera adecuada. .

Palabras Clave:

Impacto, ciencia, comunicación, traducción, gramática

\section{INTRODUCTION}

A través del tiempo, el inglés ha tomado relevancia alrededor del mundo siendo uno de los 3 más importantes. Aproximadamente
335 millones de personas hablan inglés como su primer idioma y otros 430 millones lo hablan como su segunda lengua (Katsiavriades y Qureshi, 2002), además, el inglés ha

a Giovanni Beltrán Santoyo, Centro de Trabajo Jóvenes Construyendo el Futuro área de Ciencia y Tecnología No. 91009, http://orcid.org/0000-0002-4646-0809, Email: giovannibelsan11@gmail.com

${ }^{b}$ Esther Aurora Ruíz Huerta, Universidad Nacional Autónoma de México, Instituto de Geofísica, https://orcid.org/0000-0002-6295-2221, Email: estherarh26@gmail.com

c Juan Miguel Gómez Bernal, Universidad Autónoma Metropolitana, División de Ciencias Básicas y de la Salud, https://orcid.org/00000002-8628-7967, Email: gupi02@yahoo.com.mx 
trascendido culturas y ha influenciado desde la moda hasta las tradiciones y estilos de vida (Crystal, 2004).

El inglés se utiliza ahora casi exclusivamente como lengua de la ciencia. La adopción de un lenguaje científico universal ha tenido un efecto extraordinario en la comunicación científica: al aprender un solo idioma, los científicos de todo el mundo tienen acceso a la vasta literatura científica y pueden comunicarse con otros científicos en cualquier parte del mundo. Además, hoy en día, diversas revistas indizadas de alta valía publican en este idioma, con la finalidad de agrupar a los investigadores y cohesionar más el medio de la investigación y su producción cotidiana (Drubin y Douglas, 2012; Mundo educativo, 2021).

Diferentes autores se han ocupado, desde distintos puntos de vista, del predominio de la lengua inglesa como vehículo de la ciencia en la época actual y de la influencia que ésta pueda tener sobre las diversas lenguas nacionales (Díaz, 2008; Gómez y Gómez, 2011; Drubin y Douglas, 2012; Niño-Puello, 2013; Murray-Tortarolo, 2019).

Es por eso que el propósito de este artículo es recopilar información para poder examinar y analizar críticamente los principales aspectos de la situación actual del idioma inglés dentro del campo científico y la importancia que tiene dentro de este mismo.

\section{Origen del idioma}

Independientemente de la variedad de idiomas que se están volviendo una forma de comunicación más común, el inglés ocupa su lugar como una de las formas de comunicación predominantes en el mundo y su influencia se extienden hasta más de 2000 millones de personas en todo el mundo.

El idioma inglés realmente despegó con la invasión de Gran Bretaña durante el siglo quinto. Tres tribus germánicas, los jutos, los sajones y los anglos, buscaban nuevas tierras para conquistar y cruzaron desde el Mar del Norte. Cabe señalar que el idioma inglés que conocemos y estudiamos a través de varios cursos de inglés en la actualidad aún no se había creado, ya que los habitantes de Gran Bretaña hablaban varios dialectos del idioma celta.

Durante la invasión, los británicos nativos fueron llevados al norte y al oeste hacia tierras que ahora conocemos como Escocia, Irlanda y Gales. La palabra Inglaterra y el inglés se originaron a partir de la palabra inglesa antigua Engla-land, que literalmente significa "la tierra de los anglos", donde hablaban inglés (Encyclopedia Britanica, s.f.; Oxford International s.f.).

\section{Inglés antiguo (siglos V al XI)}

Baugh y Cable (1951) notables profesores de inglés de la Universidad de Pensilvania, señalan entre los trabajos publicados en esa época que alrededor del $85 \%$ del inglés antiguo ya no está en uso; sin embargo, los elementos supervivientes forman la base del idioma inglés moderno en la actualidad (Encyclopedia Britanica, s.f.; Oxford International s.f.).

El inglés antiguo se puede subdividir en las siguientes etapas:
- $\quad$ Prehistórico o Primitivo (siglos V al VII): La literatura o documentación que haga referencia a este período no está disponible aparte de ejemplos limitados de runas anglosajonas (Stumpf, 1970). Inglés antiguo temprano (siglos VII al X): Este período contiene algunas de las primeras pruebas documentadas del idioma inglés, que muestra a autores y poetas notables como Cynewulf y Aldhelm, siendo figuras destacadas en el mundo de la literatura anglosajona.

- Inglés antiguo tardío (siglos $\mathrm{X}$ al $\mathrm{XI}$ ): Se puede considerar la fase final del idioma inglés antiguo provocada por la invasión normanda de Inglaterra. Este período terminó con la consecuente evolución del idioma inglés hacia el inglés medio temprano.

\section{Inglés medio temprano}

Durante este período el idioma inglés, y más específicamente, la gramática inglesa, comenzó a evolucionar con especial atención en la sintaxis, la cual es referida como "la disposición de palabras y frases para crear oraciones bien formadas en un idioma" (Encyclopedia Britanica, s.f.; Oxford International s.f.), y encontramos que mientras el gobierno británico y sus ciudadanos adinerados anglicanizaron el idioma, las influencias normandas y francesas siguieron siendo el idioma dominante hasta el siglo XIV.

En este período se ha atribuido a la pérdida de terminaciones de casos que, en última instancia, resultó en que los marcadores de inflexión fueran reemplazados por características más complejas del lenguaje. Las terminaciones de mayúsculas y minúsculas son "un sufijo de un sustantivo, pronombre o adjetivo declinado que indica su función gramatical" (Encyclopedia Britanica, s.f.; Oxford International s.f.).

\section{Inglés medio tardío}

Durante el siglo XIV, un dialecto diferente (conocido como East-Midlands) comenzó a desarrollarse alrededor del área de Londres. Geoffrey Chaucer, un escritor al que han llegado a identificar como el padre de la literatura inglesa y autor de los famosos Cuentos de Canterbury, fue a menudo proclamado como el mayor poeta de esa época. Fue a través de sus diversas obras que el idioma inglés fue más o menos "aprobado" junto con el francés y el latín, aunque continuó escribiendo algunos de sus caracteres en los dialectos del norte (DeMaria et al., 2013). A mediados de la década de 1400 se introdujo el estándar de inglés de la cancillería. La historia cuenta que los empleados que trabajaban para la Cancillería en Londres hablaban francés y latín con fluidez. Su trabajo era preparar documentos judiciales oficiales y, antes de la década de 1430, los dos idiomas antes mencionados eran utilizados principalmente por la realeza, iglesia y británicos adinerados. Después de esta fecha, los empleados comenzaron a usar un dialecto que sonaba de la siguiente manera (Encyclopedia Britanica, s.f.; Oxford International s.f.).

- $\quad$ gaf (gave) not yaf (Chaucer's East Midland dialect)

- $\quad$ such not switch 
theyre (their) not hir (Encyclopedia, s.f.)

\section{Inglés moderno temprano}

Los cambios en el idioma inglés durante este período ocurrieron desde el siglo XV hasta mediados del siglo XVII, y significaron no solo un cambio en la pronunciación, el vocabulario o la gramática en sí, sino también el comienzo del Renacimiento inglés, el cual tiene cimientos mucho más sólidos que su primo paneuropeo, el Renacimiento italiano, el cual surgió a finales del siglo XV. El inglés moderno temprano se asoció con el renacimiento de los movimientos sociales y culturales, y aunque tardó en cobrar fuerza durante las fases iniciales, celebró las alturas de la gloria durante la época isabelina.

La innovación de la imprenta permitió que el inglés medio moderno se convirtiera en la corriente principal. The Printing Press fue clave en la estandarización del idioma inglés mediante la distribución de la Biblia en inglés. La publicación de Caxton de Le Morte d'Arthur (La muerte de Arthur) de Thomas Malory se considera el primer bestseller de material impreso. La interpretación de Malory de varios cuentos que rodean al legendario Rey Arturo y los Caballeros de la Mesa Redonda, en sus propias palabras, y la consiguiente popularidad aseguraron indirectamente que el inglés moderno temprano llegara para quedarse.

Fue durante el reinado de Enrique VIII cuando los plebeyos ingleses finalmente pudieron leer la Biblia en un idioma que entendían, lo que en su propio grado ayudó a difundir el dialecto de la gente común.

El final del siglo XVI trajo consigo la primera traducción completa de la Biblia católica, y aunque no tuvo un impacto notable, jugó un papel importante en el desarrollo continuo del idioma inglés, especialmente con la población católica de habla inglesa mundial.

A finales del siglo XVI y principios del XVII, los escritos del actor y dramaturgo William Shakespeare conquistaron el mundo. Shakespeare comenzó a escribir durante una época en la que el idioma inglés estaba experimentando serios cambios debido al contacto con otras naciones a través de la guerra, la colonización y entre otras. Estos cambios se consolidaron aún más a través de Shakespeare y otros dramaturgos emergentes que encontraron que sus ideas no podían expresarse a través del idioma inglés actualmente en circulación. Así, la "adopción" de palabras o frases de otros idiomas se modificaron y se agregó al idioma inglés, creando una experiencia más rica para todos los involucrados.

Fue a principios del siglo XVII cuando vimos el establecimiento de la primera colonia inglesa exitosa en lo que se llamó "El Nuevo Mundo". Jamestown, Virginia, también vio el amanecer del inglés americano con colonizadores ingleses que adoptaron palabras indígenas y las agregaron al idioma inglés. La constante afluencia de sangre nueva debido a la migración voluntaria e involuntaria (es decir, esclavos) durante los siglos XVII, XVIII y XIX significó que unas variedades de dialectos ingleses cobraran vida, esto incluía influencias de África Occidental, nativos americanos, españoles y europeos (Encyclopedia Britanica, s.f.; Oxford International s.f.).

\section{Inglés moderno tardío}

La Revolución Industrial y el surgimiento del Imperio Británico durante el siglo XVIII, XIX y principios del XX vieron la expansión del idioma inglés.

Los avances y descubrimientos en ciencia y tecnología durante la Revolución Industrial vieron la necesidad de nuevas palabras, frases y conceptos para describir estas ideas e invenciones. Debido a la naturaleza de estos trabajos, los científicos y eruditos crearon palabras usando raíces griegas y latinas, por ejemplo: Bacteria, histology, nuclear, biology.

El colonialismo trajo consigo un arma de doble filo. Se puede decir que las naciones bajo el dominio del Imperio Británico vieron la introducción del idioma inglés como una forma de aprender, participar y, con suerte, beneficiarse de la influencia "extranjera". Si bien los descubrimientos científicos y tecnológicos eran algunos de los beneficios que podían compartirse, la Gran Bretaña colonial vio esto como una forma no solo de enseñar su idioma, sino de impartir su cultura y tradiciones a las sociedades que consideraban atrasadas, especialmente las de África y Asia.

La idea puede haber fracasado cuando el idioma inglés se alejó con una gran cantidad de palabras extranjeras que ahora se han convertido en parte integrante del idioma inglés, por ejemplo: shampoo, candy, cot y muchas otras que se originaron en la India (Encyclopedia Britanica, s.f.; Oxford International s.f.).

\section{EL IMPACTO DEL IDIOMA INGLÉS EN EL CAMPO CIENTÍFICO}

Los artículos de investigación científica se encuentran entre los documentos más prestigiosos producidos en la actualidad. Son los medios por los que científicos informan sobre sus contribuciones científicas. Además, la publicación de artículos de investigación es un medio para que los científicos aumenten su credibilidad (Englander, 2014). Dos de los investigadores más importantes que encabezan la investigación sobre la escritura académica para la publicación fueron, Lillis y Curry (2010), destacando el lugar del inglés en la ciencia y su papel en los procesos de globalización, incluida la difusión del conocimiento científico: "Las instituciones de prestigio son considerados por las instituciones de prestigio. Idioma de la ciencia y por muchos participantes en la producción de texto: académicos, revisores, traductores, editores de revistas científicas, como el lenguaje predeterminado de la ciencia, la investigación académica y la difusión". En la tabla 1 se comparan los idiomas más hablados durante la última década, donde podemos observar como el inglés predomina sobre los demás. 
Tabla 1: Comparación de idiomas más hablados alrededor del mundo

\begin{tabular}{|c|c|}
\hline Idioma & Hablantes \\
\hline Inglés & 1.132 millones de hablantes \\
\hline Español & 534 millones de hablantes \\
\hline Francés & 280 millones de hablantes \\
\hline Ruso & 258 millones de hablantes \\
\hline Portugués & 234 millones de hablantes \\
\hline \multicolumn{2}{|c|}{ Información obtenida de Berlitz México, $($ s.f. $)$} \\
\hline
\end{tabular}

El aumento del inglés como el lenguaje de la ciencia.

Las revistas científicas que se encargaban de difundir la naturaleza, ciencia, medicina, células y comentarios químicos en Nueva Inglaterra, se publican en inglés. De hecho, Hamel (2001) afirma ampliamente que el inglés es el lenguaje de la ciencia. Por supuesto, no siempre fue así.

En el pasado, había un idioma que se usaba para las ciencias: sumerio, griego, árabe y latín (Hamel, 2001). La "Revolución Científica" de los siglos XVI al XVIII que subyace a la actual era científica, a menudo se marca como comienzo con Nicolaus Copernicus. Escribiendo en latín, disipó la idea de que el universo rodeó la tierra en 1543, con su trabajo de Celestium del Orbium de Revolutibus (sobre las revoluciones de las esferas celestiales).

Los desarrollos comenzaron a ocurrir a un ritmo notable en los campos de las matemáticas, química, fisiología humana y física. La comunicación de los hallazgos se convirtió en parte fundamental para el esfuerzo científico. Una salida principal fueron las transacciones filosóficas de la Royal Society de Londres, establecidas en 1660, y que fueron publicadas en gran parte, pero no exclusivamente en inglés (Kaplan, 2001). A principios del siglo $\mathrm{XX}$, se utilizaron francés, alemán, ruso e inglés para comunicar la ciencia y las conferencias de ciencias multilingües fueron la norma. El alemán fue el idioma dominante de publicación de 1900 a 1920 y fue fundamental para la medicina, biología y química (Hamel, 2001). Después de la Primera Guerra Mundial, el papel de la lengua alemana se decidió, en parte porque fue prohibido en conferencias internacionales (Ammon, 2001).

La prominencia del inglés comenzó cuando el alemán se prohibió, y coincidió con el dominio de la investigación científica en los Estados Unidos. La influencia estadounidense en la ciencia y, por lo tanto, el papel del inglés también tomó tal prominencia por varias razones. En el período de posguerra, la infraestructura educativa y científica estadounidense aún estaba intacta, mientras que Europa había sido devastada por la guerra (Kaplan, 2001).

\section{El impacto de los artículos y revistas científicas}

El factor de impacto es una medida de la cantidad de veces que se cita un artículo promedio en una revista, durante un año. Clarivate Analytics publica los factores de impacto de la revista anualmente como parte de Web of Science Journal Citation Reports. Solo las revistas incluidas en Science Citation Index
Expanded (SCIE) y Social Sciences Citation Index (SSCI) reciben un factor de impacto (Elsevier Author Services, s.f.). Aunque el factor de impacto es ampliamente utilizado por instituciones e investigadores, se ha tenido una idea errónea generalizada sobre el método para calcular el factor de impacto de la revista, su importancia y cómo se puede utilizar. El sistema para determinar el impacto de una revista científica fue creado en 1961 por Eugene Garfield, fundador del Instituto de Información Científica (IIS). El IIS ahora se incorpora a la red de conocimiento de Thomson-Reuters, que incluye el Índice de Citas Ciencias enormemente importante (ICC). El factor de impacto que calcula Thomson-Reuters se ha convertido en ubicuo (Lilis y Curry, 2010). En la figura 1 uno se muestra la fórmula para calcular el factor de impacto de una revista.

\begin{tabular}{|cc|c|}
\hline $\begin{array}{l}\text { Número de citas de articulos } \\
\text { en una revista en un año } \\
\text { (por ej. 2012) }\end{array}$ & $\div \quad \begin{array}{c}\text { Número de articulos } \\
\text { publicados en esa revista } \\
\text { en los } 2 \text { años anteriores } \\
\text { (por ejemplo, 2010 y 2011) }\end{array}$
\end{tabular}$=$ Impact factor

Figura 1. Fórmula para calcular el factor de impacto de una revista

El razonamiento detrás de esta fórmula es simple: una revista publica artículos que son citados por otros; por lo tanto, la revista tiene un impacto medible en el campo (Garfield, 2006). Cuanto mayor sea el factor de impacto, mayor será el impacto. Los factores de impacto se recalculan cada año.

No obstante, el factor de impacto está ahora bien arraigado en el mundo de las publicaciones científicas. El factor de impacto se indica en las páginas web de las revistas individuales de las cuatro principales editoriales de revistas del mundo (Elsevier, Springer, Taylor \& Francis y Wiley, las cuales tienen más de 1000 revistas) (figura 2) (Ware y Mabe, 2009).

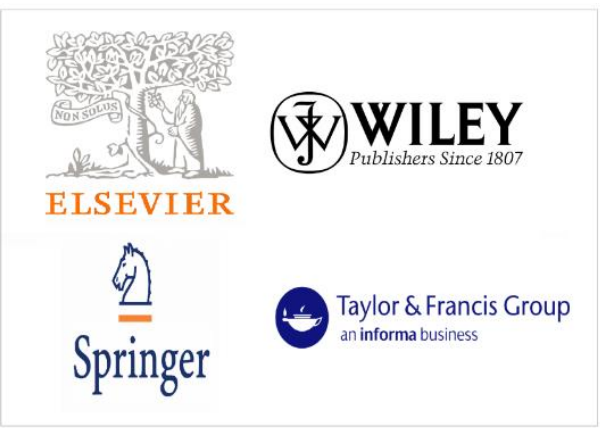

Figura 2. Principales editoriales internacionales (Logo tomado de las páginas oficiales de cada editorial)

Con el paso del tiempo, el factor de impacto ha tomado un papel muy importante como uso de calidad dentro de las revistas de carácter científico, ya que, mientras más alto sea el factor de impacto de las revistas, éstas adquieren un mayor prestigio, debido a que no muchos artículos son aceptados debido a la alta métrica. 


\section{Competencias a nivel internacional}

Debido a que el idioma inglés se ha estandarizado como el lenguaje de la ciencia, cientos de investigadores tienen como objetivo publicar sus investigaciones en una revista internacional. Aunque es deseable un alto nivel de competencia en las cuatro habilidades del inglés, la competencia de producción escrita es un componente esencial para la visibilidad en el mundo académico. El dominio de la escritura en inglés es fundamental para atraer la atención de un editor y convencer a los revisores de que acepten el manuscrito y este sea publicado. Por otro lado, para los países bajos cuya lengua materna no es el inglés, escribir manuscritos es mucho más desafiante que para los hablantes nativos de inglés, ya que los revisores critican mucho el inglés en vez de evaluar los resultados que presentan sus investigaciones (Drubin y Douglas, 2012).

En un estudio realizado en 2019 por Natural Index, se dieron a conocer los 10 países que publican más artículos científicos de manera internacional en esa editorial, entre los cuales se encuentran Estados Unidos, China, Alemania, Reino Unido, entre otros (Nature Index s.f.). En la figura 3 se muestran una gráfica de los países con más publicaciones alrededor del mundo en esa editorial.

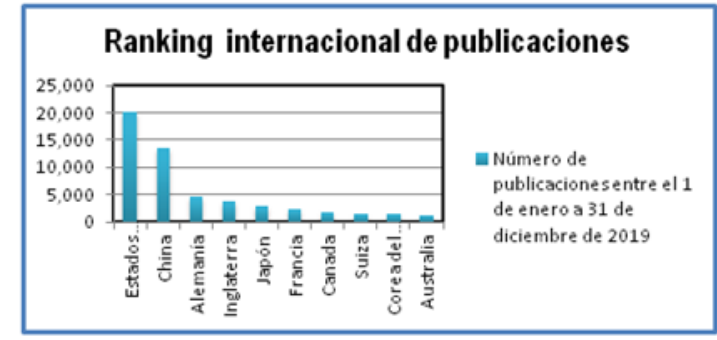

Figura 3: Ranking internacional de número de publicaciones de artículos científicos (Imagen modificada de Nature Index, s.f.)

\section{GLOBALIZACIÓN DEL INGLÉS EN LA CIENCIA}

La relación entre lenguas y globalización es tan estrecha que se llega a proponer como parte de los procesos de mundialización, la llamada "globalización de las lenguas". Dentro de algunas sociedades es común referirse al inglés como el lenguaje de la ciencia. Ya sea desde una perspectiva crítica (Phillipson, 2001; Canagarajah, 2002), o una neoliberal (Kuznetsov y Dahlman, 2008). La relación entre lenguas y globalización se está haciendo cada vez más presente en la investigación lingüística ya que la mayoría de los artículos científicos son publicados en inglés; la mayoría de los artículos publicados indexados están en inglés al igual que las bases de datos; la mayoría de las citas utilizadas en los artículos también son en inglés (Martel, 2001; Coupland, 2011; Englander, 2014).

\section{El conflicto del inglés en un nivel internacional}

La globalización debería de ser una vía para el aprendizaje de idiomas y su expansión; sin embargo, con el estatus del idioma inglés como lengua internacional, ha generado que la diversidad lingüística se haya vuelto escasa debido a la globalización que se ha formado a través de los años. Por lo que el inglés se ha transformado en la primera lengua de comunicación a nivel internacional, teniendo diversos efectos dentro del campo científico, como en los países donde el inglés no es su lengua nativa, la manera en que se comunican (ya sea verbal o escrita) es con un inglés simple y/o deficiente (Siguan, s.f.), y debido a eso, recurren a diversas herramientas para la traducción de sus textos al inglés dándoles el estilo y la gramática correcta para que estos puedan ser publicados en una revista internacional. Es importante señalar que estas estrategias y herramientas que se implementan han ayudado a evitar los principales obstáculos que tienen los autores a la hora de publicar sus trabajos, entre estos destacan, la falta de gramática y estilo (Díaz, 2008).

\section{HERRAMIENTAS LINGÜÍSTICAS EN EL CAMPO CIENTÍFICO}

Actualmente, la comunicación en el campo científico se ha expandido a un nivel internacional gracias a la globalización que ha tenido el idioma inglés a través de los años. Sin embargo, no todos los científicos dominan este idioma como segunda lengua. Este problema ha generado que la expansión no se realice de una manera global.

Es por eso que los hablantes nativos de inglés deben ofrecer comprensión, paciencia y ayuda al revisar o editar manuscritos de hablantes no nativos de inglés, y al mismo tiempo, los hablantes no nativos de inglés deben esforzarse por producir manuscritos que estén escritos con claridad (Drubin y Douglas, 2012).

Actualmente, con el gran avance que ha tenido la tecnología estos últimos años, las herramientas lingüísticas también han evolucionado, permitiendo que podamos comunicarnos en cualquier otro idioma de una manera más sencilla y práctica, permitiéndonos mejorar la gramática y estilo del idioma. Entre estas herramientas innovadoras se encuentran diversas plataformas que ayudan con el mejoramiento de las habilidades de escritura en inglés, tales como: Google Translate, Grammarly, Linguee, entre otras.

Es importante señalar que es necesario seguir los lineamientos de la editorial donde se desee publicar internacionalmente, poniendo especial atención en el estilo gramatical para tener una buena redacción y de esta manera que sea publicado alrededor del mundo.

Aunado, a esto muchas editoriales de renombre están incorporando en sus sistemas el apoyo durante todo el proceso de preparación del manuscrito, que incluye la redacción y organización del mismo, hasta asegurarse de que sea visto, leído y citado en varias revistas manteniendo el lenguaje, formato y estilo visual de cada revista (Cargill y O’Connor, 2013).

\section{CONCLUSIÓN}

Actualmente, el idioma inglés ha tenido gran influencia como medio de comunicación en distintos medios, ya que al ser una de las lenguas más utilizadas alrededor del mundo (de manera oral como escrita), este ha permitido que la comunicación haya 
adquirido una gran expansión entre países. Al mismo tiempo, ha generado un gran avance dentro del campo de la ciencia, debido a que los investigadores publican sus hallazgos en el idioma, permitiendo la difusión de la ciencia alrededor del mundo de una forma más rápida.

En esta revisión se dio a conocer como el inglés fue evolucionando y convirtiéndose en una herramienta fundamental para cualquier tipo de comunicación que se quiera transmitir. Sin embargo, algunos países que no dominan el inglés como segunda lengua tienen desventaja al intentar comunicarse a través de este idioma, ya sea publicando algún manuscrito o al leer alguna investigación. Es por eso que es importante que se introduzcan planes académicos que permitan a países que no hablan inglés prepararse de una manera adecuada y así tener las herramientas necesarias para poder dominar el idioma y dar a conocer sus investigaciones alrededor del mundo en el campo de la ciencia.

\section{REFERENCES}

[1] Ammon, U. (Ed.). (2001). The dominance of English as a language of science. Berlin: Mouton de gruyter.

[2] Baugh AC, Cable T. (1951). A History of the English Language. London: Routledge \& Kegan Paul.

[3] Berlitz Mexico (s.f) Recuperado de: berlitz.com/es-mx/blog/idiomasmas-hablados-en-el-mundo

[4] Canagarajah AS. (2002). Critical academic writing and multilingual students. Ann Arbor: MI: University of Michigan Press.

[5] Cargill M., O’Connor P. (2013). Writing scientific research articles. 2nd Edition. Wiley-Blackwell.

[6] Coupland N. (2011). The Handbook of Language and Globalization. John Wiley \& Sons.

[7] Crystal D. (2004). The Stories of English. Allen Lane. London, UK.

[8] DeMaria Jr R., Heesok C., Samantha Z. (2013). A Companion to British Literature, Volume 2: Early Modern Literature, 1450-1660. John Wiley \& Sons.

[9] Díaz MTV. (2008). El inglés como vehículo de la ciencia: influencia sobre la redacción y traducción de textos científicos. En: La traducción del futuro: mediación lingüística y cultural en el siglo XXI (pp. 471482). Promociones y Publicaciones Universitarias, PPU.

[10] Díaz MTV. (2008). El inglés como vehículo de la ciencia: influencia sobre la redacción y traducción de textos científicos. En: La traducción del futuro: mediación lingüística y cultural en el siglo XXI. Promociones y Publicaciones Universitarias, PPU. pp. 471-482

[11] Drubin David G., Douglas R.K. (2012). English as the universal language of science: opportunities and challenges. Molecular biology of the cell. 23: 1399-1399.

[12] Elsevier Author Services (s.f.) Recuperado de: https://scientificpublishing.webshop.elsevier.com/research-process/what-journalimpact-factor/

[13]Encyclopedia (s.f) Recuperado de:: http://www.encyclopedia.com/humanities/encyclopedias-almanacstranscripts-and-maps/chancery-standard

[14]Encyclopedia Britanica (s.f) Recuperado de https://www.britannica.com/

[15] Englander K. (2014). The rise of English as the language of science. Writing and Publishing Science Research Papers in English. Springer, Dordrecht.
[16] Garfield E. (2006). The history and meaning of the journal impact factor. JAMA. 295(1): 90-93.

[17] Gómez N., Gómez J I. (2011). Aproximación a una didáctica de la traducción de textos científicos y técnicos inglés-español. Íkala, revista de lenguaje y cultura. 16(27): 135-163.

[18] Hamel RE. (2001). The dominance of English in the international scientific periodical literature. AILA Review. 20. 53-71.

[19] Kaplan RB. (2001). English - the accidental language of science. Mouton de gruyter. Pp. 3-26

[20] Katsiavriades K., Qureshi T. (2002). The origin and history of the English language. Krysstal. London, 26.

[21] Kuznetsov Y., Dahlman C. (2008). Mexico's transition to a knowledgebased economy. Washington, D.C.: The World Bank.

[22] Lillis T., Curry MJ. (2010). Academic writing in a global context: The politics and practices of publishing in English. London, UK: Routledge.

[23] Martel A. (2001). 'When does knowledge have a national language? Language policy-making for science and technology'. En: U. Ammon (ed). The dominance of English as a language of science: Effects on other languages and language communities. Berlin: Mouton de Gruyter. pp. 27-58.

[24] Mundo Educativo (s.f) Recuperado de: https://www.aprendemas.com/mx/blog/mundo-educativo/comoimpacta-el-idioma-en-la-produccion-universal-de-la-ciencia-76592

[25] Murray-Tortarolo GN. (2019). De cómo escribir un artículo de divulgación y no matar de aburrimiento a tus lectores. Revista Digital Universitaria. 20(4): 1-6. doi:

http://doi.org/10.22201/codeic.16076079e.2019.v20n4.a4

[26] Nature Index(s.f) Recuperado de: https://www.nature.com/articles/d41586-020-01231-w

[27] Niño-puello M. (2013). El inglés y su importancia en la investigación científica: algunas reflexiones. Revista Colombiana de Ciencia Animal-RECIA. 5: 243-254.

[28] Oxford International (s.f) Recuperado de: https://www.oxfordinternationalenglish.com/a-brief-history-of-theenglish-language/

[29] Phillipson R. (2001). English for globalization or for the world's people?', International Review of Education. 47: 185-200.

[30] Siguan M. (s.f.). Las lenguas y la globalización. Recuperado de: http://www. euskara. euskadi. eus/contenidos/informacion/artik26_1_siguan_08_07/es _siguan/adjuntos/Miquel-Siguan-cas.

[31] Stumpf John. (1970). An Outline of English Literature; Anglo-Saxon and Middle English Literature. London: Forum House Publishing Company.

[32] Ware M., Mabe W. (2009). The STM Report: An overview of scientific and scholarly journal publishing. Oxford, UK: International Association of Scientific, Technical and Medical Publishers 